\title{
OBSERVATIONS ON BACTERIOPHAGE TYPING OF PSEUDOMONAS AERUGINOSA
}

\author{
By BOSKO POSTIC AND MAXWELL FINLAND \\ (From the Thorndike Memorial Laboratory, Second and Fourth (Harvard) Medical Services, \\ Boston City Hospital and the Department of Medicine, Harvard Medical \\ School, Boston, Mass.)
}

(Submitted for publication July 6, 1961 ; accepted July 29, 1961)

Recent reports indicate that there have been increasing numbers of serious infections due to Pseudomonas aeruginosa during the past few years (1-3). This has also been true at this hospital where the number of cases of bacteremic infection with this organism and of deaths in such cases has been steadily increasing since the introduction of potent antibacterial agents (4). Ps. aeruginosa has become one of the most important infectious agents, rivaling the staphylococcus as a cause of septicemia and death in patients with burns (5-7); it is being found frequently in infected surgical wounds (8), and outbreaks of serious and fatal infections with this organism have been reported in nurseries (9). It causes notoriously intractable urinary tract infections $(10,11)$, and it is being recognized as a major cause of serious and fatal superinfections in patients under intensive treatment with multiple or broad spectrum antibiotics (12-14). Most recently it has proved to be a major cause of bacteremia and death in patients under treatment for staphylococcal infections with methicillin (dimethoxyphenyl penicillin) (15-17). Most of the pseudomonas infections seem to arise in hospitals $(12,18,19)$ and some studies have implicated inhalation of humid air (20-22) and contaminated water (23) as sources of isolated outbreaks in nurseries, but the sources of most of the sporadic infections within hospitals remain obscure.

The use of bacteriophage typing for the characterization of strains within certain genera of bacteria is now well known and has had many applications (24). This method, applied to strains of Staphylococcus aureus has proved particularly useful in recent years for the identification of sources of endemic and epidemic spread of infections with this organism, particularly in nurseries

* Aided by Grant E-23 from the National Institutes of Health. and surgical operating rooms (25-27). However, although phages of $P$ s. aeruginosa have been recognized and studied in some detail in various laboratories, and they have been used to some extent for the classification of strains of this species and in attempts to distinguish strains from various sources, they have not been widely used for "typing" strains or in the epidemiology of pseudomonas infections in hospitals. For these reasons it seemed appropriate to explore the possible uses of pseudomonas phages for such purposes.

The present paper deals with the isolation of pseudomonas phages from clinical sources, some observations on their properties, and a preliminary exploration of the application of these and some other available phages to the typing of strains of $P$ s. aeruginosa from clinical infections. Soon after this study got under way, Gould and McLeod (28) reported in detail on their study, based on bacteriophage and serological techniques, of pseudomonas infections in hospitals. Their report, which contains the pertinent references to previous work bearing directly on this subject, has proved most valuable in the conduct of the present study.

\section{MATERIALS AND METHODS}

Bacterial strains and bacteriophages. Seven phages and their propagating strains (PS) were obtained from the American Type Culture Collection ATCC (29). The phages had been designated 12055-B1, -B2, and -B3; and $12175-\mathrm{B} 1,-\mathrm{B} 2,-\mathrm{B} 3$, and $-\mathrm{B} 4$; the propagating strain of Ps. aeruginosa for the first three phages was ATCC$12055,{ }^{1}$ and for the other four it was ATCC-12175. All other strains of $P s$. aeruginosa were isolated in the bacteriology laboratory of the Boston City Hospital and all other phages were developed from these strains in the course of this study.

The following criteria were used for identification of $P s$. aeruginosa: motile, gram-negative rods ; typical colonial morphology on 10 per cent sheep's blood agar and on eosin methylene blue agar; for most, but not all,

\footnotetext{
1 This is host C10 of Dickinson and Codd $(30,31)$.
} 
strains a diffusible green, or, in a few, a reddish-brown pigment on trypticase soybean agar; pellicle formation in liquid media; growth at 30 to $42^{\circ} \mathrm{C}$; glucose the only one of the commonly-used sugars fermented; and characteristic odor. In a previous study of pseudomonas strains reported from this laboratory (32), the formation of a green or brown color on Gessard's glycerol agar was found to be the most reliable test for the production of pyocyanin; by that criterion 103 of 110 strains were classified as $P$ s. aeruginosa and 7 were considered to be atypical strains of pseudomonas, since they failed to produce the pigment. No such differentiation was attempted in the present study.

In all, 161 strains of $P$ s. aeruginosa were isolated and used in this study. Most of them were obtained from cultures of urine or infected wounds, some from blood, peritoneal exudate or cerebrospinal fluid, and occasional ones from specimens of feces that were negative for enteric pathogens and in which the predominating coliform bacilli had first been suppressed by growth in liquid tetrathionate medium.

Isolation, propagation and characterization of $P s$. aeruginosa bacteriophages. The procedures were similar in many respects to those used by Gould and McLeod (28). The media used routinely were: trypticase soy broth $^{2}$ (TSB), 1.5 per cent trypticase soy agar ${ }^{2}$ (TSA), and a semisolid medium consisting of equal parts TSB and TSA.

Each of the phages obtained from ATCC was propagated as follows: $1 \mathrm{ml}$ of a 5-hour TSB culture of the propagating strain was flooded on a TSA plate and the excess fluid removed. The plate was placed in an incubator at $37^{\circ} \mathrm{C}$ with the lid tilted for 25 minutes to permit drying, after which $1 \mathrm{ml}$ of concentrated phage suspension containing $10^{6}$ to $10^{8}$ phage particles was poured over the surface and permitted to dry again in the same manner after removing the excess fluid. The plate was covered and incubated overnight (16 to 18 hours) at $31^{\circ} \mathrm{C}$. The surface of the plate then appeared to be largely iree of the bacterial lawn and had only isolated colonies, which usually represented phage-resistant or lysogenic mutants. The "bare" plate was refrigerated at $-20^{\circ}$ to $-30^{\circ} \mathrm{C}$ for 24 to 72 hours and then thawed at room temperature, yielding varying amounts of clear fluid containing concentrated phage, which was then harvested as the clear supernatant after centrifugation at $3,000 \mathrm{rpm}$ for 30 to 60 minutes. The phage suspension was heated at $58^{\circ}$ to $60^{\circ} \mathrm{C}$ for 2 hours to kill any residual bacterial cells. This procedure did not affect the titer of phage appreciably, thus confirming the observations of Dickinson (33).

The titer of phage in the suspension was estimated by plaque counting (24) and also roughly by delivering single drops of 10 -fold dilutions of the suspension with a Pasteur pipet on a dried lawn of the propagating strain. The plaque-counting method also permitted visualization of plaque morphology. The routine test dilution (RTD) for that phage suspension was then selected. This differed from that employed in phage typing of staphylo-

2 Obtained from Baltimore Biological Laboratories. cocci (34) in that 10 or, more often, 100 times the highest dilution giving confluent lysis was used as the RTD for the present study in order to permit visualization of various types of lysis produced by the same phage on different strains; this also served to reduce to some extent the variations in the degrees of susceptibility of different strains of Ps. aeruginosa to any given phage. The RTD for most of the phages was a $10^{-2}$ dilution of the phage suspension, but it ranged from $10^{-1}$ to $10^{-4}$ (equivalent to about $10^{4}$ to $10^{8}$ or more phage particles per $\mathrm{ml}$ by the plaque-counting method).

In the isolation of new phages, advantage was taken of the spontaneous lysis observed in some cultures of $P s$. aeruginosa on solid media as evidenced by the moth-eaten appearance of individual colonies, probably representing an unstable lysogenic state of that culture (temperate phage). ${ }^{3}$ An isolated moth-eaten colony together with its base agar was cut out and put into TSB, which was then refrigerated for 48 or 72 hours at $4^{\circ} \mathrm{C}$ and centrifuged. The supernatant was removed, heated, and used as a phage suspension. A lawn of each strain of $P s$. aeruginosa to be tested was then prepared on the surface of an agar plate and individual drops of the various phage suspensions were placed on this lawn. The susceptibility of the bacterial strain to these phages was evidenced by the development of clear plaques. Bacterial strains showing confluent lysis with any phage suspension were then chosen as indicator and propagating strain.* When a suitable host or indicator strain was not found in this manner, a bacterial lawn was flooded with the phage suspension and isolated clear plaques were then looked for as representing virulent phage. Phage mutants which had a wider host-range than their parent phages were sometimes derived in this manner. Each isolated plaque was then picked and placed in a growing 4-hour broth culture of the bacterial strain which had yielded the plaque. The mixture was incubated for only 6 hours, at which time the maximal yield of phage was obtained. The suspension of the lysed areas was then refrigerated and subsequently subjected to centrifugation, heating, titration, and propagation on agar plates as already described for the known phages.

The "phage typing" of strains of pseudomonas was carried out essentially as with staphylococci, using a lawn of a 5-hour TSB culture on "checkerboard" plates on which a drop of RTD (as defined above) of each of the phages was placed. The plates were read after overnight incubation. Clear, confluent lysis was read as,+++ 20 to 30 clear plaques as ++ , less than 20 clear plaques as + , and no lysis as - . Another type of reproducible lysis, best described as "turbid plaque" formation was also re-

3 The "metallic spots" sometimes noted in pseudomonas cultures are morphologically different and probably do not represent a phage phenomenon (35).

4 An "indicator" bacterial strain is one that is lysed by a particular phage but not by some others; a "propogating" strain is one that is maintained and used to prepare a stock of a particular phage (and sometimes more than one) to which it is susceptible. 
corded and designated by the addition of " $T$ " after the phage designation. The type or pattern of a pseudomonas strain was then represented by the designation of the phages which produced confluent, that is, +++ or $+++\mathrm{T}$ lysis.

The visualization and counting of plaques was done with double-layered TSA plates. The base layer contained $10 \mathrm{ml}$ TSA (1.5 per cent), and the surface layer consisted of $2 \mathrm{ml}$ of semisolid agar ( 0.75 per cent TSA) containing $0.1 \mathrm{ml}$ of the desired dilution of phage suspension and $0.1 \mathrm{ml}$ of a 5 -hour TSB culture of the propagating strain. The soft agar hardened promptly after being delivered over the previously hardened base layer. The number and morphology of the plaques were recorded after overnight incubation.

Bacterial mutants resistant to a particular phage were developed from a susceptible bacterial strain by flooding a suspension of phage on a lawn of the culture and allowing it to grow overnight. Individual phage-resistant colonies were picked into TSB and grown overnight at $37^{\circ} \mathrm{C}$. The resulting culture was washed in TSB, diluted, and plated so as to produce individual colonies. A culture from a single colony was retyped and then used as an indicator strain in the plaque visualization and counting procedure for the phage in question in order to ascertain the nature and degree of the resistance of the mutant strain to that phage.

\section{RESULTS}

Development of the set of typing phages. Three criteria were used in selecting phages for inclusion in typing set: 1) host range, as determined by exposure of the 161 isolates from this hospital and the 2 host strains obtained from ATCC;2) development of resistance to individual phages and tests of cross resistance to other phages; and 3) visualization of plaque morphology. When two or more preparations of phage suspensions derived from different sources behaved in identical manner with respect to these three criteria, only one of them was chosen for inclusion in the set together with the propagating strain that gave the best yield of that phage. The 13 distinct phages thus chosen are listed in Table I, which also lists the relevant data concerning their sources as well as those of their respective propagating strains. The phages were divided into four groups, based on similarities with respect to one or more of the three criteria. The lytic patterns produced by these phages on all of the propagating strains are shown in Table II.

Phage typing of clinical isolates of Ps. aeruginosa. The set of 13 phages thus selected was used in the "phage typing" of 161 isolates of Ps. aeruginosa obtained from various clinical sources throughout the hospital. The largest number, 49, were from infected urines; 25 from infected wounds ; 22 from sputum or tracheal aspirates; 14 from cultures of feces which, as already noted, had been found negative for enteric pathogens and from which the predominant coliform organisms had been eliminated by cultivation in tetrathionate

TABLE I

The set of Pseudomonas aeruginosa typing phages

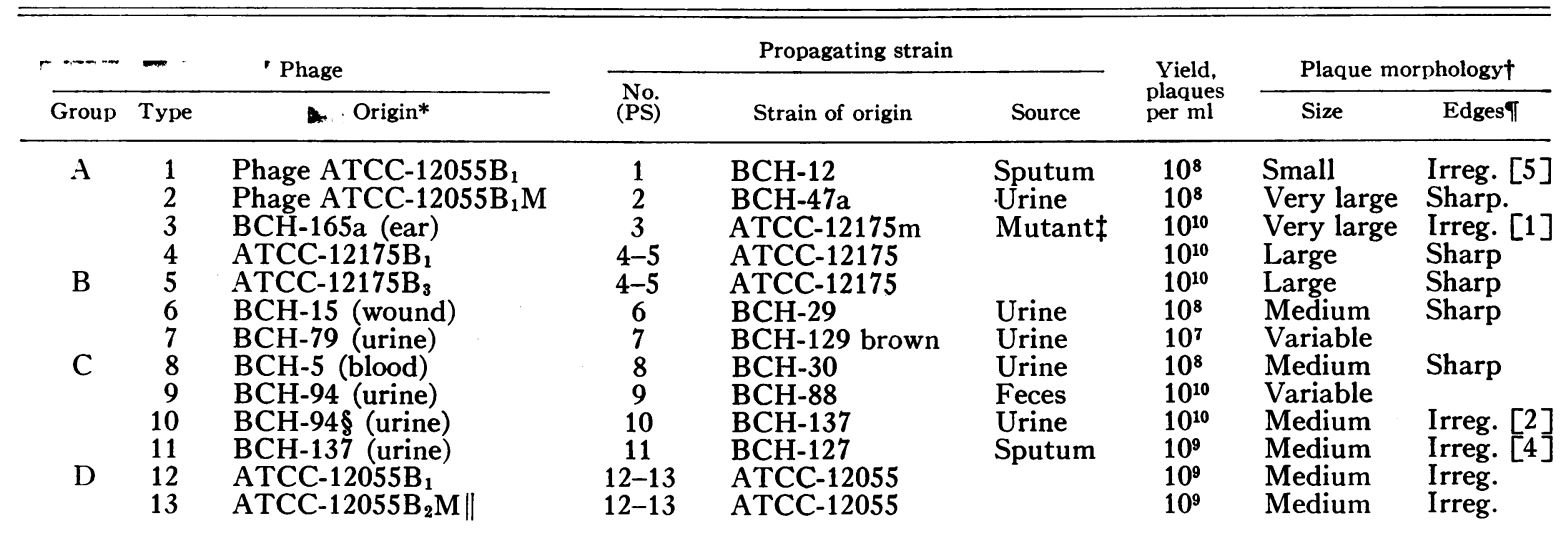

* Source of phage or original designation of the lysogenic donor strain of Ps. aeruginosa, the source of which is given in parentheses.

$\dagger$ All plaques were clear.

T See Figure [ ].

$\ddagger$ Mutant bacterial strain selected for resistance to phage $12175 \mathrm{~B}_{3}$ (which is here designated as phage 5).

$\S$ Mutant phage isolated from the same lysogenic strain of $P s$. aeruginosa that yielded phage 9.

I| This mutant phage produced clear confluent lysis. 
TABLE II

Pattern of lysis of propagating strains (PS) by the set of typing phages

\begin{tabular}{|c|c|c|c|c|c|c|c|c|c|c|c|c|c|c|}
\hline \multirow{3}{*}{$\begin{array}{l}\text { No. } \\
\text { (PS) }\end{array}$} & \multicolumn{13}{|c|}{ Phages } & \multirow{3}{*}{$\begin{array}{c}\text { Phage } \\
\text { pattern* }\end{array}$} \\
\hline & \multicolumn{4}{|c|}{ A } & \multicolumn{3}{|c|}{ B } & \multicolumn{4}{|c|}{ C } & \multicolumn{2}{|c|}{ D } & \\
\hline & 1 & 2 & 3 & 4 & 5 & 6 & 7 & 8 & 9 & 10 & 11 & 12 & 13 & \\
\hline 1 & +++ & + & \pm & \pm & - & - & - & - & - & - & - & - & - & $A(1)$ \\
\hline 2 & & & $=$ & $=$ & + & - & - & - & - & - & - & - & - & $A(2)$ \\
\hline 3 & $+++\mathrm{T} t$ & $+++T$ & ++ & $++t$ & \pm & - & - & - & - & - & - & - & - & $\mathrm{A}(3 / 4)$ \\
\hline $4-5$ & $++t+$ & $++t T$ & ++ & $++t$ & ++ & $++t$ & - & - & - & - & - & - & - & $\mathrm{A} / \mathrm{B}(3 / 4 / 5 / 6)$ \\
\hline 6 & $+\dot{t}+\mathrm{T}$ & + & + & ++ & + & $t+\dot{t}$ & - & - & - & - & - & - & - & $\mathrm{B}(6)$ \\
\hline 7 & $=$ & - & - & - & $+\bar{t}+$ & $+\bar{t}+$ & +++ & $\overline{\overline{1}}+$ & - & - & - & - & - & \\
\hline $\begin{array}{l}8 \\
9\end{array}$ & $\overline{-}$ & $\bar{z}$ & $\overline{-}$ & $\overline{-}$ & + \pm+ & + \pm+ & $\overline{-}$ & 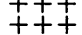 & $+\overline{+}+$ & $+\bar{t}+$ & $\bar{z}$ & $\bar{z}$ & $\overline{-}$ & $\begin{array}{l}\mathrm{B} / \mathrm{C}(5 / 6 / 8) \\
\mathrm{C}(8 / 9 / 10)\end{array}$ \\
\hline 10 & - & - & - & - & - & - & - & - & + & $+t$ & - & - & - & $\mathrm{C}(10)$ \\
\hline 11 & - & $++t T$ & - & - & - & - & - & - & - & - & +++ & - & - & C(11) \\
\hline $12-13$ & + & & - & + & - & - & - & - & - & - & - & +++ & $++t$ & $\mathrm{D}(12 / 13)$ \\
\hline
\end{tabular}

* Pattern based only on confluent $(+++)$, complete (clear) lysis

$t \mathrm{~T}=$ turbid or hazy lysis, not listed in the phage pattern.

broth; 10 from otitic exudate; 6 from blood cultures; and the rest from other or unknown sources. The latter included 20 strains that had been isolated 18 or more months previously for different studies and had been kept frozen, whereas all of the others were recent isolates made after this study began.

The strains were then classified according to the patterns of lysis with RTD of the different phages, separating those on which the lysis was confluent and clear from those yielding only turbid lysis. In every instance in which confluent lysis, clear or turbid, was produced with RTD (as here defined) of any given phage, the same type of lysis was reproduced on subcultures of the same strain with multiples of RTD. Both the clear and turbid lyses were reproduced on different occasions at least once, and the latter was reproduced numerous times with two strains. Moreover, the same pattern of both kinds of lysis was reproduced whenever multiple isolates of $P$ s. aeruginosa were obtained from the same or different sources in the same patient. A third type of lysis was observed with phage 7 when it was applied undiluted on certain strains. This was an irregular pattern of lysis with fuzzy edges which was also reproducible with subcultures of the same strains.

The results of the phage typing of the 161 isolates are summarized in Table III, which also lists the sources of the strains of each phage pattern. From part $A$ of this table it is seen that 78 strains (48.4 per cent) could be typed on the basis of the patterns of clear and confluent lysis, and that 19 phage patterns of such lysis were discerned among the 78 strains. Included among the 78 were 2 to
4 isolates obtained from the same or different sources in each of seven patients. In each of these patients all of the isolates produced the identical phage pattern; in three this was $B(5 / 6)$; in three others it was $B(5 / 7)$; and the isolates from the seventh patient all typed at $B(7)$, each producing the same type of reddish-brown pigment. The proportion of strains from each source (i.e., from urines, wounds, and so forth) that could be typed on the basis of clear lysis did not differ markedly from that for the total of all strains except that a somewhat smaller proportion of those of miscellaneous or unknown sources could be typed in this manner. Of the 29 strains in the latter category, only 9 (31 per cent) produced patterns of clear lysis.

Part B of Table III lists the number of isolates from different sources that failed to produce confluent clear lysis but which yielded reproducible patterns of uniformly turbid lysis with RTD of some of these typing phages. There were 50 such strains and it is interesting to note that all of them reacted only with RTD of the four phages classified here as group A. Also listed as of the last phage pattern in part B of Table III are 16 strains that yielded irregular plaques of turbid lysis with fuzzy edges only with the undiluted suspension of phage 7. A total of 65 strains (40.4 per cent) could be typed in this manner, and they fell into nine patterns, including $\mathrm{B}(7 \mathrm{~T})$. Multiple isolates of Ps. aeruginosa were obtained at different times from the same or different sources in four patients; here again all isolates from any one patient yielded the same phage pattern.

In all therefore, 143 , or 88.8 per cent, of the 161 
TABLE III

"Phage patterns" and sources of 161 clinical isolates of Ps. aeruginosa

\begin{tabular}{|c|c|c|c|c|c|c|c|c|c|}
\hline \multirow[b]{2}{*}{ Phage pattern } & \multicolumn{9}{|c|}{ Number of strains isolated from: } \\
\hline & $\begin{array}{l}\text { Infected } \\
\text { urine }\end{array}$ & $\begin{array}{l}\text { Infected } \\
\text { wounds }\end{array}$ & $\begin{array}{l}\text { Sputum } \\
\text { or } \\
\text { tracheal } \\
\text { exudate }\end{array}$ & Feces $^{a}$ & $\begin{array}{c}\text { Otitic } \\
\text { exudate }\end{array}$ & $\begin{array}{c}\text { Blood } \\
\text { culture }\end{array}$ & $\begin{array}{c}\text { Deep } \\
\text { abscess }\end{array}$ & $\begin{array}{l}\text { Others, } \\
\text { or } \\
\text { unknown } \\
\text { source }\end{array}$ & $\begin{array}{c}\text { All } \\
\text { sources }\end{array}$ \\
\hline
\end{tabular}

A. Patterns of confluent clear lysis with RTD

$\mathrm{A}(1)$
$\mathrm{A}(1 / 2)$
$\mathrm{A}(2)$
$\mathrm{A}(2 / 3 / 4)$
$\mathrm{B}(5)$
$\mathrm{B}(5 / 6)$
$\mathrm{B}(5 / 7)$
$\mathrm{B}(6)$
$\mathrm{B}(7)$
$\mathrm{B} / \mathrm{C}(5 / 8)$
$\mathrm{B} / \mathrm{C}(5 / 6 / 8)$
$\mathrm{B} / \mathrm{C}(7 / 8)$
$\mathrm{C}(8)$
$\mathrm{C}(9)$
$\mathrm{C}(10)$
$\mathrm{C}(11)$
$\mathrm{C}(8 / 9 / 10)$
$\mathrm{C}(8 / 9 / 10 / 11)$
$\mathrm{C}(9 / 10)$

Total

$\mathrm{A}(1 \mathrm{~T})$

$\mathrm{A}(1 \mathrm{~T} / 2 \mathrm{~T})$

$\mathrm{A}(1 \mathrm{~T} / 2 \mathrm{~T} / 3 \mathrm{~T})$

$\mathrm{A}(1 \mathrm{~T} / 2 \mathrm{~T} / 4 \mathrm{~T})$

$\mathrm{A}(1 \mathrm{~T} / 4 \mathrm{~T})$

$\mathrm{A}(1 \mathrm{~T} / 2 \mathrm{~T} / 3 \mathrm{~T} / 4 \mathrm{~T})$

$\mathrm{A}(2 \mathrm{~T})$

$\mathrm{A}(4 \mathrm{~T})$

$\mathrm{B}(7 \mathrm{~T})^{\mathrm{h}}$

Total

NT

All strains
1

1

2
6
3

1

1

1
1
2

23

B. Patterns of confluent but turbid or hazy lysis with RTD

\begin{tabular}{|c|c|c|c|c|c|c|c|c|}
\hline $\begin{array}{l}2 \\
3\end{array}$ & 3 & 4 & 4 & 3 & & & $\begin{array}{l}1 \\
1^{f}\end{array}$ & $\begin{array}{c}5 \\
20 \mathrm{~g}\end{array}$ \\
\hline 1 & & 1 & & & & & 4 & $\begin{array}{l}1 \\
5\end{array}$ \\
\hline 5 & 1 & & & & & & 1 & 7 \\
\hline & 1 & & & 1 & & & 4 & 6 \\
\hline 1 & 1 & & 1 & & 1 & & 1 & $\begin{array}{l}2 \\
3\end{array}$ \\
\hline 8 & 1 & 2 & & & 1 & 1 & 3 & $16^{i}$ \\
\hline 20 & 9 & 7 & 5 & 4 & 2 & 3 & 15 & 65 \\
\hline & & & ith $\mathrm{R}$ & or $\mathrm{cc}$ & atec & & & \\
\hline $\begin{array}{r}6 \\
49\end{array}$ & 25 & $\begin{array}{r}1 \\
22\end{array}$ & $\begin{array}{r}1 \\
14\end{array}$ & $\begin{array}{r}2 \\
10\end{array}$ & $\begin{array}{l}1 \\
6\end{array}$ & $\begin{array}{l}2 \\
6\end{array}$ & $\begin{array}{r}5 \\
29\end{array}$ & $\begin{array}{c}18^{\mathrm{j}} \\
161\end{array}$ \\
\hline
\end{tabular}

a All fecal strains were from specimens negative for enteric pathogens.

b Four separate isolates ( 3 from leg ulcer and 1 from urine) from one patient; 3 separate isolates ( 2 ear and 1 throat culture) from a second patient and 2 (both from wound) from a third.

- Two separate isolates from each of 3 patients (both from urine in one, both from sputum in the second and $1 \mathrm{from}$ urine and the other from a blood culture in the third).

d All from separate urines obtained at different times from the same patient and each produced reddish-brown pigment.

One from osteomyelitis, 1 from empyema.

${ }^{\mathrm{f}}$ From cerebrospinal fluid.

s Two strains isolated from separate stools of same patient.

h Irregular plaques of turbid or hazy lysis produced only with undiluted phage.

i Four separate isolates from one patient (1 each from urine, nephrostomy drainage, blood culture and cerebrospinal fluid); 2 isolates from different urine specimens in a second patient and 2 isolates (one from sputum and the other from a burn wound) in a third.

$j$ Two of these were separate isolates from an abdominal abscess in one patient and 2 others were from otitic exudate in a second patient. 
TABLE IV

Susceptibility of parent and phage-resistant mutant strains of Ps. aeruginosa to the set of typing phages

\begin{tabular}{|c|c|c|c|c|c|c|c|c|c|c|c|c|c|c|}
\hline \multirow{3}{*}{$\begin{array}{l}\text { Strain of } P s . \\
\text { aeruginosa* }\end{array}$} & \multicolumn{13}{|c|}{ Phages } & \multirow{3}{*}{$\begin{array}{c}\text { Phage } \\
\text { pattern† }\end{array}$} \\
\hline & \multicolumn{4}{|c|}{ A } & \multicolumn{3}{|c|}{ B } & \multicolumn{4}{|c|}{$\mathrm{C}$} & \multicolumn{2}{|c|}{$\mathrm{D}$} & \\
\hline & 1 & 2 & 3 & 4 & 5 & 6 & 7 & 8 & 9 & 10 & 11 & 12 & 13 & \\
\hline $\begin{array}{l}\text { BCH-63 Parent } \\
\text { Mutant }\end{array}$ & $\begin{array}{l}+++T \\
++t\end{array}$ & $\begin{array}{l}+++\mathrm{T} \\
+++\mathrm{T}\end{array}$ & $\overline{-}$ & $\overline{-}$ & $\begin{array}{l}+++ \\
++\end{array}$ & $\overline{-}$ & $+\underline{+}$ & $\overline{-}$ & $\overline{-}$ & $\overline{-}$ & $\overline{-}$ & $\overline{-}$ & $\overline{-}$ & $\begin{array}{l}\mathrm{B}(5 / 7) \\
\mathrm{B}(5)\end{array}$ \\
\hline $\begin{array}{l}\text { BCH-31 Parent } \\
\text { Mutant\& }\end{array}$ & $=$ & $=$ & $\overline{-}$ & $\overline{-}$ & $\overline{-}$ & $\overline{-}$ & $\overline{-}$ & $+\underline{+}$ & $\begin{array}{l}+t+ \\
++t\end{array}$ & $\begin{array}{l}+t+ \\
+t+\end{array}$ & $\overline{-}$ & - & $\overline{-}$ & $\begin{array}{l}\mathrm{C}(8 / 9 / 10) \\
(\mathrm{C} 9 / 10)\end{array}$ \\
\hline
\end{tabular}

* Another such pair of strains, shown in Table II, consists of PS4-5 (parent) and PS3 (mutant resistant to phages 5 and 6).

† Confluent, clear lysis.

\$ Resistant to phage 8 .

strains could be classified with these phages. The remaining 18 strains ( 11.2 per cent) failed to produce any lysis with any of the 13 phages of the typing set and were therefore classified as nontypable (NT), as shown in part C of Table III.

Not shown in Table III is the distribution of the patients from whom the strains were obtained among the different hospital services and wards. Actually, the strains of each of the phage patterns were obtained from many wards and there was no concentration of these patients in any given part of the hospital. Three was the largest number of patients with strains of the same phage pattern on any one ward; they were among the 15 patients from whom strains of $\mathrm{B}(5 / 6)$ were isolated. There were four wards in which two patients yielded strains of the same phage pattern, a different phage pattern being represented in each ward. ${ }^{5}$

Development of phage-resistant mutants from phage-susceptible bacterial strains. In the course of this study, isolated colonies of bacterial growth were sometimes observed within an area of otherwise clear and confluent lysis produced on the lawn of a strain of Ps. aeruginosa on which one of the phage suspensions had been placed. Some of these colonies were subcultured, washed free of residual phage and studied for their susceptibility to the phages of the typing set. The results thus obtained with two mutant bacterial strains, resistant to the respective phages by which they were recognized, with their respective parent strains are shown in Table IV. A third pair of such strains was included among the propagating strains of the typing set of phages, namely, the

5 These pairs of patients were, respectively, from among 7 patients with type $\mathrm{B}(6) ; 15$ with $\mathrm{B}(5 / 7) ; 4$ with $\mathrm{C}(8 / 9 / 10)$; and 19 with phage pattern $\mathrm{A}(1 \mathrm{~T} / 2 \mathrm{~T})$. parent strain PS4-5 and the derived mutant PS3 isolated from the area of lysis of PS4-5 produced with phage 5 .

From Table IV it is seen that the mutant bacterial strain derived from $\mathrm{BCH}-63$ after it had been acted on by phage 7 gave a phage pattern identical with that of the parent strain except for its failure to be lysed by that phage. Likewise, the phage pattern of the mutant derived from $\mathrm{BCH}-31$ when that strain reacted with phage 8 was identical with its parent except for its resistance to phage 8. From Table II it is seen that PS3 which was derived from a colony within the area of lysis produced on PS4 5 by phage 5 was partially resistant to phage 5 and completely resistant to phage 6 , to which PS4-5 was fully sensitive.

Observations on the color produced by some strains of $P$ s. aeruginosa and their phage susceptibility. Phage 7 of the present typing set is characterized by a rather wide host range. Among the 161 clinical isolates, it produced clear and confluent lysis in RDT of 23 strains, and irregular and atypical lysis of 16 others when applied as the undiluted suspension, each type of lysis being reproducible in every instance. The action of phage 7 on the propagating strains for the typing set of phages, however, was highly specific, as shown in Table II. Only its own PS (BCH129), which regularly produced a diffusible reddish-brown pigment on TSA, was fully susceptible to phage 7. The development of this phage is of interest.

Early in the present study it was noted that strains yielding a diffusible brownish-red or brownto-purple color [probably due to pyorubrin (36)] were not susceptible to any of the phages originally obtained from ATCC. Only a phage derived 
from the lysogenic yield of a clinical strain of Ps. aeruginosa, $\mathrm{BCH}-79$, when propagated on another strain, $\mathrm{BCH}-63$, produced a few plaques of lysis on three available strains that produced deep brown pigment, viz:, BCH-90, -98 and -129 . These three isolates had all been obtained from cultures of separate urine specimens of one patient with chronic pyelonephritis. The plaques produced on $\mathrm{BCH}-129$ were then propagated first in a growing broth culture of that bacterial strain, the heated supernatant was further propagated on TSA plates seeded with $\mathrm{BCH}-129$ which was then selected as PS7, and the resulting phage was included in the typing set as phage 7 .

The sequence of events and probable mechanisms involved were somewhat as follows. The original phage yield from the brown, lysogenic strain $\mathrm{BCH}-79$, when propagated on the green strain $\mathrm{BCH}-63$, contained a phage mutant with a wider host range which included the brown isolates $\mathrm{BCH}-90,-98$ and -129 . After propagation on the brown strain BCH-129, now PS7, the phage (now labeled phage 7 ) retained its wide host range irrespective of whether it was propagated on the green BCH-63 or the brown PS7. Furthermore, the concentrated suspension of phage 7 could lyse several strains of pseudomonas not susceptible to other phages and some of these strains yielded a diffusible purplish color on TSA, especially if the growth was allowed to stand for

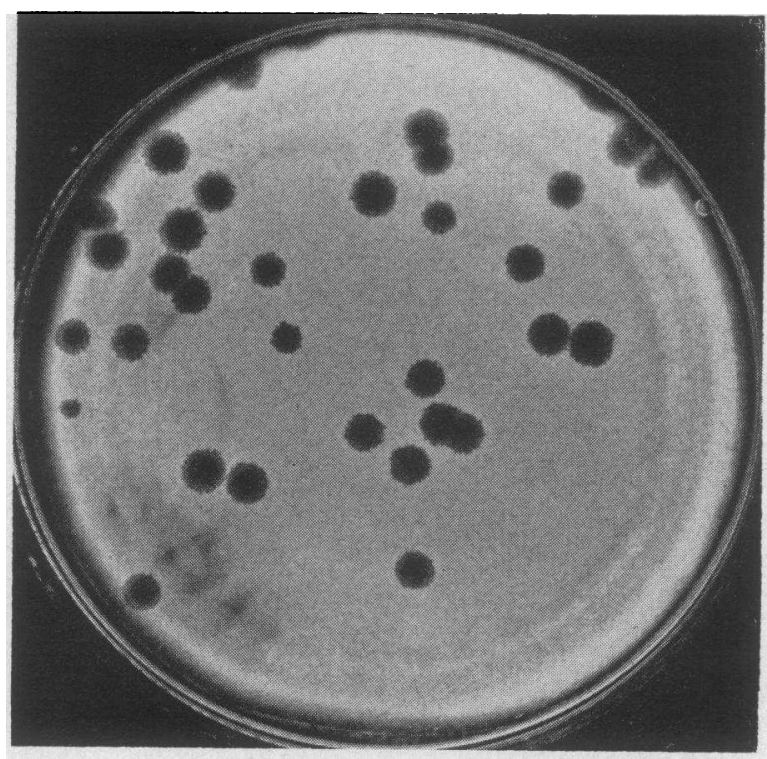

Fig. 1. Dilution $10^{-8}$ of Phage 3 on PS3. The plaques are clear, very large and have irregular edges.

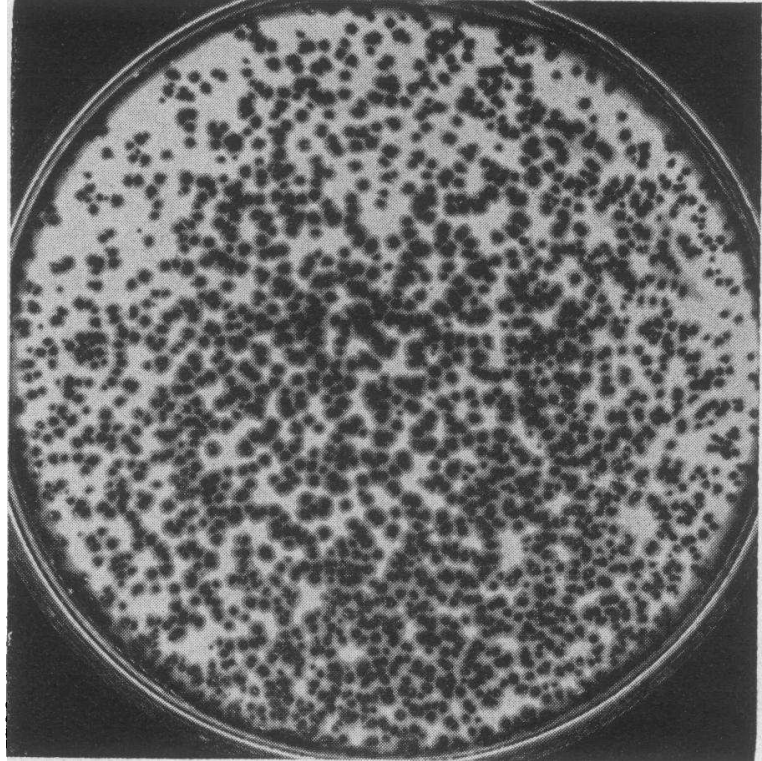

Fig. 2. Dilution $10^{-7}$ of Phage 10 on PS10. The plaques are clear, of medium size, and sharply defined but of irregular outline.

1 or 2 days exposed to daylight. Three such strains were $\mathrm{BCH}-19,-20$ and -27 . However, although the concentrated phage 7 produced irregular lysis, and individual plaques of varying size could be demonstrated by the plaque-visualization method, repeated attempts to propagate phage from these plaques in growing cultures of strains $\mathrm{BCH}-19,-20$ and -27 failed. It therefore seems highly probable that the irregular lysis was the result of a direct lethal effect of the large numbers of phage particles on the bacterial strains.

The loss of color production frequently observed in some strains of Ps. aeruginosa $(32,37)$ makes the estimation of the yield of pigment an unstable genetic marker for these organisms. The ability of phage 7 to lyse both green and brown (red) strains is therefore of some interest.

Observations on plaque morphology. The final separation of phage clones was carried out by the plaque-counting technique which also permitted visualization of the morphology of the individual plaques. Figures 1 and 2 illustrate the appearance of the plaques of two of these phages.

The turbid ${ }^{6}$ plaque was of particular interest and the subject of more detailed study because it

${ }^{6}$ The term is used here to describe the centeral lytic area of the plaque and does not refer to the peripheral zones of partial lysis, such as those shown in Figures 4 and 5. 


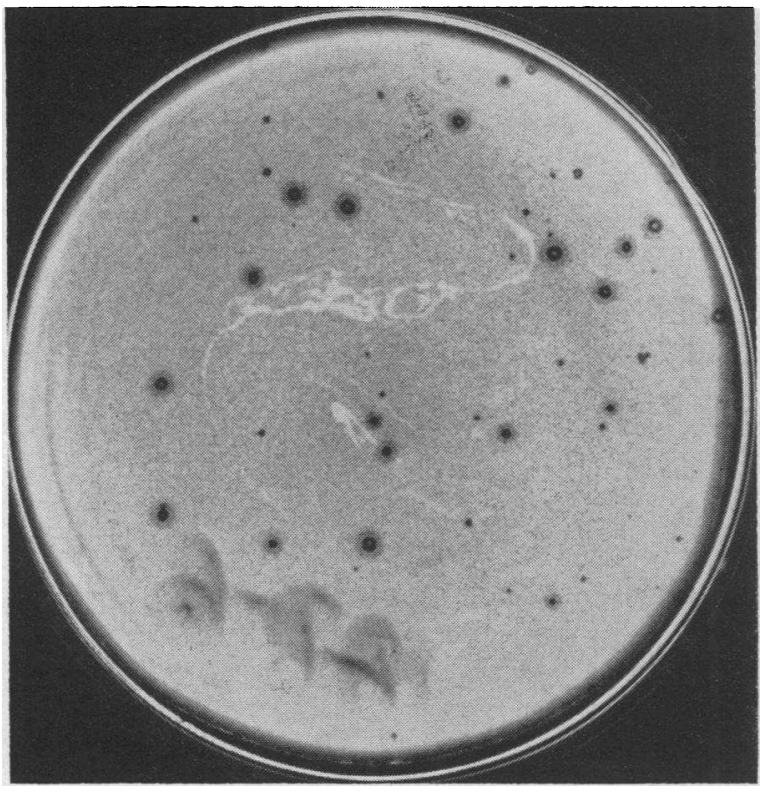

Fig. 3. Dilution $10^{-7}$ of Phage 11 as originally isoLATED FROM THE PHAGE YIELD OF BCH-127 PROPAGATED ON THAT STRAIN AS AN INDICATOR. Note the three types of plaques: clear center with irregular edges; turbid center within a clear area and irregular outer borders; very small clear plaques. A fourth type, small and diffusely turbid, was also present but not seen in the photograph.

was frequently employed, under special conditions, in the typing of strains of pseudomonas, as already noted. Several possibilities were considered to explain this variety of plaque morphology: 1) mixed host bacterial culture; 2) lysogenic variants or phage-resistant mutants selected out of the bacterial culture during exposure to a specific phage; 3) a mixture of phages; 4) development of host-range mutants of the phage; and 5) temperate phage with lysogenization of part of the host culture exposed to the phage and lysis of the rest of the culture by virulent phage.

An example of an attempt to analyze the mechanism of turbid plaque formation may be cited from the experience with the early development of typing phage 11. The initial phage suspension from which this phage was derived produced a mixture of clear and turbid plaques on the indicator strain that was used, as shown in Figure 3. When one of the clear plaques was picked and propagated on the same host culture, it gave rise to clear plaques exclusively, as shown in Figure 4.

To rule out the possibility that a mixed culture accounted for the turbid plaque, PS11 was grown in TSB and then 10 indivdual colonies were picked from TSA pour plates of end-dilution of that culture. Each colony was grown separately in broth and then tested with the original phage suspension. Both clear and turbid plaques were produced on the cultures from each of the 10 colonies in a manner similar to that depicted in Figure 3. However, when purified phage suspensions were prepared by propagating single clear plaques on each of these single colony cultures, only clear plaques resulted, and these were always reproducible. Likewise, phage suspensions derived from single turbid plaques prepared in the same manner always yielded turbid plaques when propagated on each of the single colony cultures. This seems to indicate that the differences in plaque morphology were not the result of a mixed host culture.

When a drop of RTD of a suspension of phage prepared from one of the turbid plaques was used on a lawn of PS11, it produced confluent turbid lysis. A turbid section of agar was cut out of such a plate, inoculated into TSB, and a number of individual colony cultures prepared, as above. Each of the resulting cultures was then tested separately for susceptibility to the clear plaqueforming phage and to the phage prepared from the turbid plaque. All were found to be resistant to both varieties of phage. On the other hand,

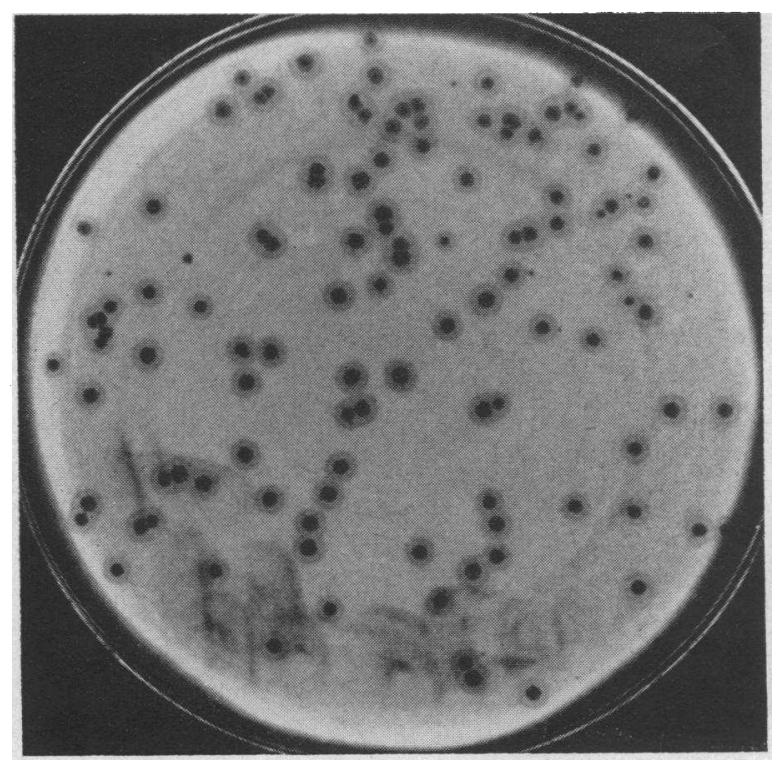

Fig. 4. Dilution $10^{-6}$ of the Clear PlaQUe variant SELECTED aS PHAGE 11 When GROWN WITH PS11. 
when the clear plaques were cultured in TSB, they yielded no growth, indicating the presence of virulent phage exclusively, which lysed all of the bacterial cells within the clear plaque.

This phenomenon of hazy plaque formation was noted by Dickinson (31) with her host strain C-10 of Ps. pyocyanea which served as PS for phages ATCC-12055- $B_{1},-B_{2}$ and $-B_{3}$. She concluded that the clear plaque-forming phage was a virulent mutant of a predominantly temperate phage yield of a lysogenic strain of pseudomonas. The temperate phage, on the other hand, produced hazy plaques capable of establishing a lysogenic state in the host (indicator) strain.

Turbid plaques could also be produced artificially with a single virulent phage suspension screened against a background of a mixture of phage-sensitive and phage-resistant bacteria. Thus, when phage 7 , which is lytic for $P s$. aeruginosa strain $\mathrm{BCH}-63$ was used to infect a bacterial lawn consisting of a mixture of $\mathrm{BCH}-63$ and a phage 7-resistant mutant of that strain (Table IV), confluent turbid plaques resulted. Normally, phage 7 produced only clear plaques on the susceptible indicator strain, $\mathrm{BCH}-63$, and had no effect on the phage 7-resistant mutant of that strain.

In the present study some of the typing phages

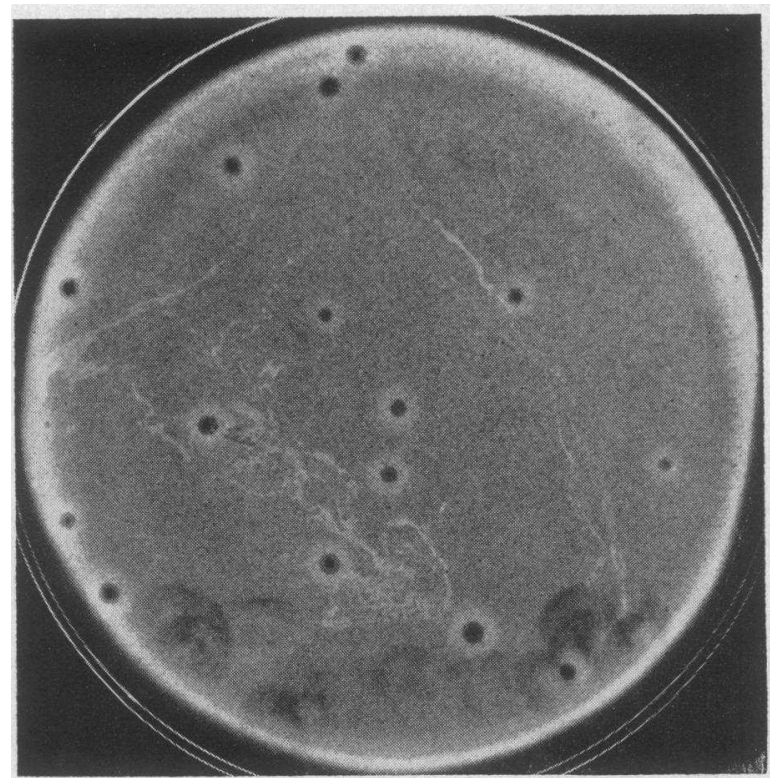

Fig. 5. Dilution $10^{-8}$ of Phage 1 on PS1. Note the clear centers of the plaques and their irregular edges.

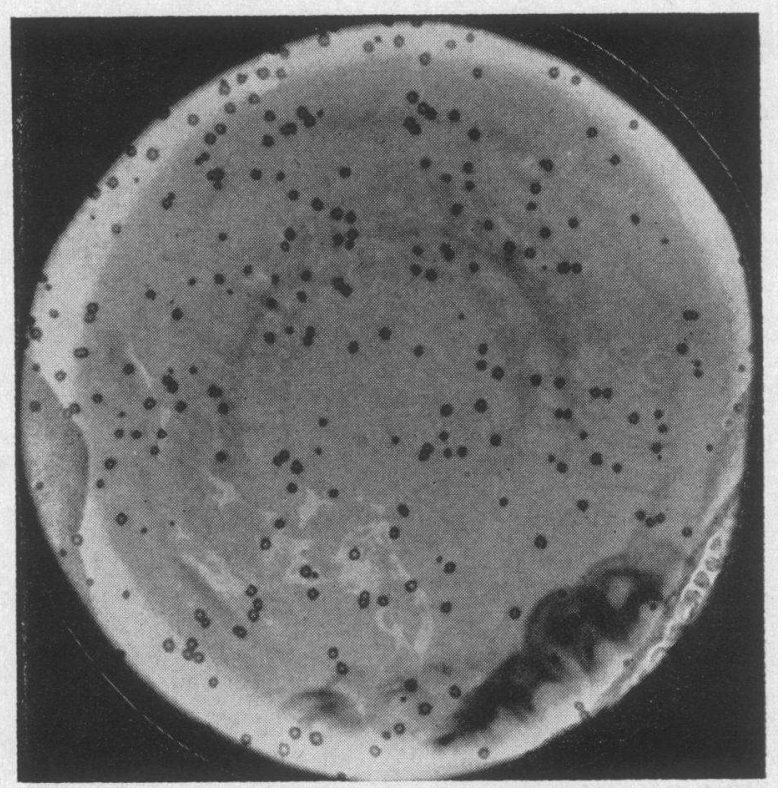

Fig. 6. Dilution $10^{-6}$ of Phage 1 on Indicator Strain $\mathrm{BCH}-138$. Note the turbid centers of the plaques and compare them with the clear plaques produced by this phage on PS1 (Fig. 5).

produced confluent clear plaques on their own propagating strains and on very few other strains, but they produced almost exclusively turbid plaques on all other susceptible bacterial host cultures. This was the case with phage 1 which produced clear lysis on PS1, as shown in Figure 5, and on only one other clinical strain of Ps. aeruginosa, whereas it produced only turbid plaques on a large number of hospital strains of pseudomonas (Table III) as illustrated in Figure 6. This is probably the result of the different degrees of virulence of phage 1 for the different bacterial strains. Phages 2, 3 and 4 also behaved in the same manner as phage 1 , as can be seen from parts A and B of Table III. The frequent and reproducible finding of turbid plaque formation in the typing of clinical isolates of $P s$. aeruginosa by the set of typing phages, when used in concentrations greater than the minimum that produced confluent lysis on the PS, was the reason for selecting RTD in the manner used in this study.

In the isolation of new phages, when a lysogenic bacterial strain serving as a phage donor was screened alone on semisolid agar, no plaques were visualized. This was to be expected, since only a certain proportion of the bacterial cells of the moth-eaten colony would lyse and yield phage to 
which the remaining cells were presumably resistant. If the moth-eaten colony were incubated in broth, the phage-resistant bacteria would multiply without an increase in the yield of phage. For that reason, refrigeration at below freezing temperatures, followed by thawing of the suspension of the unstable lysogenic phage donor colony was used rather than incubation, as recommended by Gould and McLeod (28).

\section{DISCUSSION}

The present study should be considered only as an exploration of the possibility of utilizing bacteriophage for the classification of human pathogenic strains of $P$ s. aeruginosa and for the possible use of phage typing in the epidemiology of serious infections caused by this species. From that point of view the study seems to have been successful. It has been possible, with relative ease, to apply some of the basic phage technology and the rather limited previous data available on the application of bacteriophage to clinical strains of this organism, to establish a set of typing phages and to apply them in a manner somewhat similar to, but not identical with that used in phage typing of Staphylococcus aureus. Thus far the clinical virulence of the strains does not seem to be correlated with the phage types.

Most of the phages in the typing set were developed in the course of this study and only four of them were used directly from other sources or adapted from previously known phages. Those developed here have not yet been compared with those developed and used in other laboratories $(28,38-41)$. Moreover, it is recognized that phages of pseudomonas, as well demonstrated in this study, may be rather difficult to maintain in "pure" lines because of the frequency of lysogenization and the unstable lysogenic state of the bacterial hosts. However, the possibility of "purifying" the phages by the methods applied here seems feasible so that it is reasonable to look forward to a system of phage typing not much less satisfactory than that now available for Staphylococcus aureus.

Several favorable and promising aspects were brought out in this study. Among them is the fact that once the possibility of a variant or mixture of phage or culture was recognized, it proved feasible in most instances to select and purify the phage and its propagating strain so that they were again useful for typing purposes. Even more important is the fact that typing of any given clinical strain was reproducible with the same cultures and with subsequent subcultures. Moreover, in every instance in which multiple isolates of $P$ s.aeruginosa were made at the same time from different sources or at different times from the same or different sources in the same patient, all of them produced a phage pattern essentially identical with the typing set. This suggests that the organisms retain their specific pattern of phage susceptibility in any given patient, a property which is essential if typing is to be useful in clinical and epidemiological work. Finally, it is most encouraging that nearly 90 per cent of 161 strains isolated from clinical materials in this hospital over a period of several months could be classified with the typing set that was developed.

It remains to be determined to what extent the typing set can be maintained in reasonably stable form over long periods. It will also be necessary to determine how widely these phages can be applied in the identification of strains from other sources within this hospital and from patients and other sources in other hospitals or outside of hospitals. The stability of the individual phages and the susceptibility of bacterial strains under various types of storage and for prolonged periods must also be studied.

\section{SUMMARY AND CONCLUSIONS}

The method used in the isolation, purification and characterization of bacteriophages for Pseudomonas aeruginosa has been presented. A set of 13 distinct phages was separated on the basis of host range, plaque morphology, mutation and cross resistance. These phages were employed in the classification of 161 strains of Ps. aeruginosa isolated from clinical sources in this hospital. It was possible to classify or type 88.8 per cent of these bacterial strains on the basis of the patterns of lysis produced by this set of typing phages. Some observations are presented on the relation of phage susceptibility to the reddish-brown color of some strains of $P$ s. aeruginosa. Studies on the significance of turbid plaque formation and its use in typing are also described.

The reproducibility of the phage patterns with any given strain and the similarity of the phage 
pattern of different isolates from the same patient suggest that phage typing of Ps. aeruginosa may prove useful for epidemiological purposes.

\section{ACKNOWLEDGMENTS}

The authors are indebted to A. Kathleen Daly and Alice McDonald of the Bacteriology Laboratory, Mallory Institute of Pathology, for the original isolation of the strains of pseudomonas used in this study. They are particularly grateful to Dr. Gösta Wallmark and Dr. Harold Amos for helpful suggestions on the conduct of the study and to the latter also for assistance in the preparation of the manuscript. The ATCC phages and propagating strains were furnished by Dr. Leonard Berman from the Bacteriological Research Laboratory of Dr. Edward H. Kass. Technical assistance was rendered by Clare Wilcox and Joan Yarrows.

\section{REFERENCES}

1. Yow, E. M. Development of proteus and pseudomonas infections during antibiotic therapy. J. Amer. med. Ass. 1952, 149, 1184.

2. Erwin, C. P., Waisbren, B. A., and Kruse, R. Clinical and laboratory studies of infections due to Pseudomonas aeruginosa and pseudomonas species. Amer. J. med. Sci. 1953, 226, 525.

3. Asay, L. D., and Koch, R. Pseudomonas infections in infants and children. New Engl. J. Med. 1960, 262, 1062.

4. Finland, M. Treatment of pneumonia and other serious infections. New Engl. J. Med. 1960, 263, 207.

5. Graber, C. D., Tumbusch, W. T., and Vogel, E. H., Jr. In vitro sensitivity of pseudomonads from burned patients to colistin sulfate. Antibiot. Ann. 19591960, 7, 77.

6. Lowbury, E. J. L. Infection of burns. Brit. med. J. 1960, $1,994$.

7. Markley, K., Gurmendi, G., Chavez, P. M., and Bazan, A. Fatal pseudomonas septicemias in burned patients. Ann. Surg. 1957, 145, 175.

8. Sussman, M., and Stevens, J. Pseudomonas pyocyanea wound infection: An outbreak in an orthopaedic unit. Lancet 1960, 2, 734.

9. Burns, R. P., and Rhodes, D. H., Jr. Pseudomonas eye infection as cause of death in premature infants. Arch. Ophthal. (Chicago) 1961, 65, 517.

10. Kass, E. H. Symposium on Newer Aspects of Antibiotics: Chemotherapeutic and Antibiotic Drugs in Management of Infections of Urinary Tract. Amer. J. Med. 1955, 18, 764.

11. Murphy, G. P., and McDonald, D. F. Proteus, pseudomonas and achromobacter septicemias as complications of chronic infection in anatomically predisposed urinary tracts. J. Urol. (Baltimore) $1961,85,672$.
12. Curtin, J. A., Petersdorf, R. G., and Bennett, I. L., Jr. Pseudomonas bacteremia: Review of ninetyone cases. Ann. intern. Med. 1961, 54, 1077.

13. Forkner, C. E., Jr., Frei, E., III, Edgcomb, J. H., and Utz, J. P. Pseudomonas septicemia; observations on twenty-three cases. Amer. J. Med. 1958, 25, 877.

14. Williams, R., Williams, E. D., and Hyams, D. E. Cross-infection with Pseudomonas pyocyanea. Lancet 1960, 1, 376.

15. Smith, I. M., and Counts, G. W. Laboratory and clinical studies of dimethoxyphenyl penicillin in Symposium on the New Dimethoxyphenyl Penicillin, P. A. Bunn, Ed. Syracuse, State Univ. of New York, 1961, pp. 83-92.

16. Hewitt, W. L., and others. Clinical experience with dimethoxyphenyl penicillin in Symposium on the New Dimethoxyphenyl Penicillin, P. A. Bunn, Ed. Syracuse, State Univ. of New York, 1961, pp. 127145.

17. Yow, E. M., and Nassar, H. A. Report on the laboratory and clinical evaluation of dimethoxyphenyl penicillin in Symposium on the New Dimethoxyphenyl Penicillin, P. A. Bunn, Ed. Syracuse, State Univ. of New York, 1961, pp. 183-193.

18. Pyrah, L. N., Goldie, W., Parsons, F. M., and Raper, F. P. Control of Pseudomonas pyocyanea infection in a urological ward. Lancet 1955, 2, 314.

19. McLeod, J. W. The hospital urine bottle and bedpan as reservoirs of infection by Pseudomonas pyocyanea. Lancet 1958, 1, 394.

20. Hoffman, M. A., and Finberg, L. Pseudomonas infections in infants associated with high-humidity environments. J. Pediat. 1955, 46, 626.

21. Jørgensen, S., Dons, N., and Eriksen, K. R. Humidified oxygen as a source of infection. Anaesthesia 1959, 14, 409.

22. Trimble, G. X. Pseudomonas infections (correspondence). New Engl. J. Med. 1960, 263, 103.

23. Wilson, M. G., Nelson, R. C., Phillips, L. H., and Boak, R. A. New source of Pseudomonas aeruginosa in a nursery. J. Amer. med. Ass. 1961, 175, 1146.

24. Adams, M. H. Bacteriophages. New York, Interscience Publishers, 1959.

25. Blair, J. E., and Carr, M. Staphylococci in hospital-acquired infections; types encountered in the United States. J. Amer. med. Ass. 1958, 166, 1192.

26. Williams, R. E. O. Epidemic staphylococci. Lancet 1959, 1, 190.

27. Blair, J. E., and Carr, M. Distribution of phage groups of Staphylococcus aureus in the years 1927 through 1947. Science 1960, 132, 1247.

28. Gould, J. C., and McLeod, J. W. A study of the use of agglutinating sera and phage lysis in the classification of strains of Pseudomonas aeruginosa. J. Path. Bact. 1960, 79, 295.

29. Catalogue of Cultures, 6th ed. Washington, D. C., American Type Culture Collection, 1958, p. 85. 
30. Dickinson, L., and Codd, S. The bacteriophages of Pseudomonas pyocyanea. 2. Bacteriophage reproduction in an iridescent strain. J. gen. Microbiol. $1952,6,1$.

31. Dickinson, L. The behaviour of a temperate phage of Pseudomonas aeruginosa compared with that of a serologically related, virulent mutant. J. gen. Microbiol. 1954, 11, 105.

32. Wright, S. S., Potee, K. G., and Finland, M. Susceptibility of pseudomonas to ten antibiotics in vitro; some properties of recently isolated strains. Amer. J. clin. Path. 1954, 24, 1121.

33. Dickinson, L. The bacteriophages of Pseudomonas pyocyanea. 1. The effect of various substances upon their development. J. gen. Microbiol. 1948, 2, 154.

34. Williams, R. E. O., and Rippon, J. E. Bacteriophage typing of Staphylococcus aureus. J. Hyg. (London) 1952, 50, 320.

35. Conge, G. Taches métalliques de $B$. pyocyaneus et bactériophages. Ann. Inst. Pasteur 1948, 75, 368.
36. King, E. O., Ward, M. K., and Raney, D. E. Two simple media for the demonstration of pyocyanin and fluorescin. J. Lab. clin. Med. 1954, 44, 301.

37. Liu, P. V. Identification of pathogenic pseudomonads by extracellular antigens. J. Bact. 1961, 81, 28.

38. Don, P. A., and van den Ende, M. A preliminary study of bacteriophages of Pseudomonas aeruginosa. J. Hyg. (London). 1950, 48, 196.

39. Van den Ende, M., Don, P. A., Elford, W. J., Challice, C. E., Dawson, I. M., and Hotchin, J. E. The bacteriophages of Pseudomonas aeruginosa; filtration measurements and electron microscopy. J. Hyg. (London) 1952, 50, 12.

40. Holloway, B. W. Grouping Pseudomonas aeruginosa by lysogenicity and pyocinogenicity. J. Path. Bact. 1960, 80, 448.

41. Graber, C. D., Vogel, E. H., Jr., Brame, R., and Drake, C. H. Phagetype specificity of pseudomonads isolated from burned patients (abstract). Bact. Proc. 1961, p. 128. 